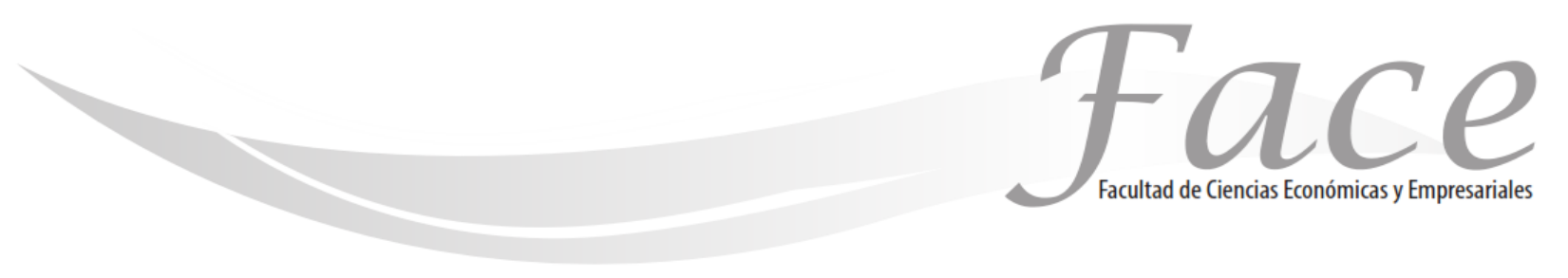

ISSN 1794-9920

Volumen $15-\mathrm{N}^{\circ} 2$

Año 2015

Págs. 17 - 28

\title{
EL FÚTBOL COMO VEHÍCULO COMERCIAL DE MARCAS DE BIENES Y SERVICIOS CASO: CLUB DEPORTES TOLIMA *
}

Andrés Darío Hoyos Arango **

Fecha de Recepción: 24 de Octubre 2015

Fecha de Aprobación: 2 de Diciembre 2015

\section{Resumen:}

El presente artículo tiene como propósito analizar diferentes factores que permitan el desarrollo de un plan de marketing deportivo dirigido al Club Deportes Tolima, a fin de lograr el posicionamiento de su marca como vehículo comercial de otras marcas, bienes y servicios nacionales e internacionales. Para establecer estos factores se diseñó una investigación de tipo cualitativo con las técnicas de análisis de documentos y entrevistas en profundidad. Dado que la falta de un plan de marketing a largo plazo, junto a la falta de apoyo de las empresas privadas regionales y nacionales, hace que en la actualidad sea un club con una fanaticada reducida y subexplotado económicamente.

Palabras clave: Marketing Deportivo, Posicionamiento, Vehículo comercial, Investigación cualitativa. Club Deportes Tolima.

\footnotetext{
*Artículo resultado Tesis de Maestría en Dirección de Marketing.

** Profesional en Mercadeo y Negocios Internacionales. Magíster en Dirección de Marketing. Docente del programa de Publicidad -Universidad Pontificia Bolivariana, Seccional Palmira-Colombia .Email: andresd.hoyos@upb.edu.co
} 


\title{
Face \\ FOOTBALL AS COMMERCIAL VEHICLE BRANDS OF GOODS AND SERVICES. CASE: CLUB DEPORTES TOLIMA
}

\begin{abstract}
Abstact:
This article has as purpose analyze the factors that allow the development of a sports marketing plan aimed to the Club Sports Tolima, to achieve the positioning of your brand as a commercial vehicle for other brands, goods and services national and international. To establish these factors was designed a type of qualitative research with the techniques of analysis of documents and in-depth interviews. Given that the lack of a marketing plan in the long term, along with the lack of support from the private companies such as national regional, means there is now a club with a small fanbase.
\end{abstract}

Keywords: Sports Marketing, Positioning, commercial vehicles, Qualitative research, Club Deportes Tolima.

\section{FUTEBOL COMO VEÍCULO COMERCIAIS DE MARCAS DE BENS E SERVIÇOS. CASO: CLUB DEPORTES TOLIMA}

\author{
Resumo:
}

Este artigo tem por objetivo analisar os fatores que permitem o desenvolvimento de um plano de marketing esportivo direccionado ao Clube Deportes Tolima, para alcançar o posicionamento da marca como veículo comercial, bens e serviços nacionais e internacionais. Para definir estes fatores se faz pesquisa com técnicas qualitativas de análise de documentos e entrevistas em profundidade. Uma vez que a falta de um plano de marketing de longo prazo, juntamente com a falta de apoio de empresas privadas regionais e nacionais, faz hoje seja um clube com uma pequena base de fãs.

Palavras-chave: Sports Marketing, Posicionamento, veículos comerciais, pesquisa qualitativa. Sport Club Tolima. 


\section{EL FÚTBOL COMO VEHÍCULO COMERCIAL DE MARCAS DE BIENES Y SERVICIOS CASO: CLUB DEPORTES TOLIMA}

Andrés Darío Hoyos Arango

\section{INTRODUCCIÓN:}

Son varios los clubes de fútbol, pertenecientes a la División Mayor del Fútbol Colombiano (Dimayor), que muestran serios déficits en sus balances desde el año 2008; de acuerdo con el informe de resultados presentado al Instituto Colombiano del Deporte, Coldeportes, el 31 de diciembre de 2009; donde se expuso una cifra de 11,7 millones de dólares (21.320 millones de pesos colombianos) en saldo negativo. Tan sólo cinco clubes (Deportes Quindío, Deportivo Pereira, Deportivo Pasto, Atlético Huila y Santa $\mathrm{Fe}$ ) obtuvieron utilidades en esa temporada, ratificando, una vez más el mal momento que atravesaban entonces y aún mantienen algunas instituciones futbolísticas en Colombia (Jiménez, 2011).

En medio de esta situación, el equipo de fútbol Club Deportes Tolima, se ha mostrado como un club regular desde el año 2003, logrando varios reconocimientos en las clasificaciones de los torneos tanto locales, como internacionales (Copa Libertadores y Copa Sudamericana); no obstante, el club presentaba, al 2009, un déficit de 6,8 millones de dólares (1.243 millones de pesos colombianos). Esta situación continuó avanzando hasta que en el mes de noviembre del 2011 sus balances financieros arrojaron pérdidas (Jiménez, 2011). Esta sonada crisis deja en evidencia que en nuestro país, en pleno siglo XXI, no se percibe el deporte como industria.

Posteriormente, con el anuncio que hace Bavaria de patrocinar al equipo solo con 200 millones de pesos por dos meses 0 un año según se ubicara la publicidad en el pecho 0 las mangas de las camisetas; sumando a que el patrocinio de la gobernación de Tolima se daría luego del segundo semestre y sería de 360 millones de pesos, la problemática se intensificó, puesto que "Gabriel Camargo, máximo accionista del equipo, aseguró que necesita 2.3 millones de dólares (4.200 millones de pesos) para que el equipo actúe" (Gómez, 2009, p.1).

Finalmente, las empresas patrocinadoras, Bavaria y Cemex, le retiraron los fondos al club. Para Ricardo Salazar (Gerente del club): "el equipo se viene asfixiando hace más de una década, los aportes que llegan son de buena voluntad pero no alcanzan. Los ingresos por venta de jugadores se acabaron y las taquillas se vinieron al piso (...)" (Torres, 2009, s.p.)

Para enfrentar esas crisis la venta de jugadores salvaba la situación, pero lo que más afectó al club fue el retiro del patrocinador principal Bavaria (Torres, 2009). Al respecto Rodríguez (2009), manifestaba la inconformidad de los hinchas, ante la falta de una propuesta sería de marketing que permitiera recursos sólidos y la explotación de la marca a fin de garantizar sus sostenimiento.

Aun con estas dificultades, el panorama puede mejorar para el 2015 y 2016, si se recupera la cartera vigente, por concepto de cuentas por cobrar de la venta de jugadores y publicidad. Según el gerente deportivo del club, se viene concretando el apoyo económico por parte del gobierno municipal de lbagué, el préstamo de jugadores y la consolidación de jugadores juveniles que podrían, en un futuro cercano, participar en el fútbol internacional y la aparición de manera lenta de nuevos patrocinadores mientras el equipo ha tenido regularidad en el campeonato de los años 2010, 2011, 2012, 2013 y 2014 donde fue protagonista de la liga.

Es evidente que este tipo de situaciones no se habrían presentado de existir un buen plan de marketing deportivo, que asumiera que los deportes y los clubes no necesitan empresas patrocinadoras sino socios; como lo sostiene Molina \& Aguiar (2003), consultor experto en Marketing Deportivo; quien agrega que "a un equipo hay que verlo como una marca, tal y como lo hacen aquellos de la talla del Manchester United o el Real Madrid, por citar solo dos ejemplos del fútbol mundial." (p.12), en este documento se expone entonces el estudio de los factores que permitirían a este club explotar la marca y permitirle ser canal para la comercialización de marcas, bienes y servicios.

\section{MARCO TEÓRICO:}

\section{Competitividad:}

Teniendo en cuenta lo expuesto por Porter (1990), para quien el valor de la competitividad está en satisfacer 0 rebasar las necesidades de los clientes y hacerlo de manera eficiente. Toda organización precisa de una estrategia para ofrecer valor superior a sus clientes. La capacidad que tenga una empresa de crearlo y obtener rentabilidad en el mercado, con relación a sus competidores, mediante el uso apropiado de los inputs para producir bienes y servicios valiosos, es lo que se denomina competitividad. Para ello, introduce cuatro facetas que componen el rombo de la productividad de Porter (Diamante de Porter): las condiciones de los factores, las condiciones de la demanda, el contexto para la estrategia y la rivalidad y las industrias afines $y$ auxiliares. 
En este sentido, se puede obtener ventaja competitiva debido a la información continua sobre necesidades y procesos, a través de innovación e inversión, detectando y explotando ventajas en los segmentos que no estén totalmente servidos junto a características nuevas para los productos. Para llevar esto a cabo, es indispensable tener en cuenta los aspectos internos de la empresa, como elemento constitutivo del diamante $\mathrm{y}$, así, generar la diferenciación como factor de competitividad y unidad estratégica (Porter, 1990). Otras definiciones importantes en cuanto al tema de competitividad son:

\section{Los Factores de Competitividad:}

En la economía moderna se enmarcan los efectos de la rentabilidad del mercado y el estudio de las tres unidades de análisis estratégico: Industria, Empresa y Grupo. Porter (citado por González, 2010) hace referencia a este último concepto como el "Grupo Estratégico"; o los diferentes grupos que puede llegar a constituir una empresa. A su vez, Tallman \& Atchinson (citado por González, 2010), lo definen como un conjunto de empresas cuya configuración estratégica es similar, con productos que ocupan posiciones similares en el mercado; organización interna similar, donde se persiguen las mismas rentas económicas con recursos similares. La importancia del grupo estratégico radica en obtener las herramientas analíticas que permitan segmentar las industrias en conjuntos de empresas cuyos competidores, accionistas y resultados sean relevantes entre sí (González, 2010).

\section{Cadenas de Valor y Canales de Marketing:}

Kotler y Keller (2006), se refieren a la ventaja competitiva, como la capacidad de una empresa para lograr resultados que, de una u otra manera, sus competidores no pueden alcanzar. En este sentido, las industrias no sólo deben tener ventajas competitivas absolutas frente a los clientes, sino además relativas frente a la competencia, acompañada de costos bajos. En ese orden de ideas, una cadena de valor es el centro del sistema de alianzas y colaboraciones que crean las empresas para generar, mejorar y entregar sus ofertas; mediante "la inclusión de actores directos e indirectos, los cuales organizan para ofrecer un valor superior al mercado meta" (Kotler \& Keller, 2006, p. 267).

Los canales de marketing, por su parte, son conjuntos de organizaciones interdependientes que participan en el proceso de poner a disposición de los consumidores un bien o servicio para su uso 0 adquisición. Los intermediarios de mercado (mayoristas y minoristas), agentes 0 corredores y proveedores de servicios 0 facilitadores (transportadores, almacenes, bancos etc.), son considerados como canales de marketing. (Kotler \& Keller, 2006).

\section{Asociatividad:}

En términos de la cadena de valor, la asociatividad es la herramienta que facilita la integración empresarial; es el mecanismo de cooperación entre empresas pequeñas y medianas, en donde cada participante, manteniendo su independencia jurídica y autonomía gerencial, decide participar en un esfuerzo conjunto con los otros participantes para la búsqueda de un objetivo común (López, 2003).

Estas modalidades de cooperación se pueden dar mediante redes horizontales (empresas que se sitúan en posiciones distintas y consecutivas de la cadena productiva, las cuales se asocian para alcanzar ventajas competitivas que no podrían obtener de forma individual) y redes verticales (alianzas entre empresas que ofrecen el mismo producto o servicio, para cooperar entre sí en algunas actividades, sin dejar de competir en un mismo mercado).

\section{Creación de Valor:}

En el proceso de generación y entrega de valor, las empresas deben primero seleccionar el valor (la tarea de marketing que se deberá realizar antes de que exista cualquier producto); luego, generar valor, que es determinar las características específicas del producto, precio y distribución y; finalmente, comunicar el valor, haciendo uso de la promoción, publicidad y demás herramientas de comunicación para dar a conocer el producto (Kotler \& Keller, 2006).

\section{Marketing:}

Dentro de un contexto de negocios, el marketing es un sistema total de actividades comerciales que tienen el propósito de planear, fijar precios, promover y distribuir productos satisfactores de necesidades, a los consumidores 0 usuarios, para alcanzar los objetivos organizacionales. La diferencia principal entre el marketing y la venta, es que en la venta el interés se centra en el producto; en el marketing, se centra en las necesidades y deseos del cliente (Kotler, 2001). Su objetivo central es conocer y entender al consumidor, de forma tal que los productos o servicios se ajusten perfectamente a sus necesidades y se vendan solos (Drucker, 2004). Así, el "Marketing es un sistema total de actividades de negocios cuya finalidad es planear, fijar el precio, promover y distribuir productos, satisfactores de necesidades entre los mercados meta para alcanzar los objetivos corporativos" (Stanton, 1994, p.11). 


\section{EL FÚTBOL COMO VEHÍCULO COMERCIAL DE MARCAS DE BIENES Y SERVICIOS CASO: CLUB DEPORTES TOLIMA}

\section{Posicionamiento de Marca:}

Para Kotler \& Keller, (2006), el posicionamiento se define como "la acción de diseñar la oferta y la imagen de un producto, servicio o empresa, de tal modo que ocupe un lugar distintivo en la mente del consumidor."(p.12). Un posicionamiento de marca adecuado sirve de directriz para la estrategia de marketing, puesto que transmite la esencia de la marca, y comunica los beneficios que obtienen los consumidores con el producto o servicio.

\section{Definición de Marca:}

De acuerdo con Wells, Moriarty \& Burnett (2007), la marca es el nombre, término, diseño o símbolo que identifica a los bienes, servicios, instituciones o ideas que postula un vendedor. Pero lo más importante es considerar la percepción que se genera del producto, en este caso, el Club debe afrontar una elección de la manera como quiere ser visto. No es una sorpresa que en la actualidad la marca vale como herramienta de marketing; logrando instalarse en el cliente, que la puede asociar a beneficios, siempre que se haya logrado un respaldo y fidelización. (Kotler, 2002).

\section{Alcance de las marcas:}

Si se tiene en cuenta que desarrollar una marca, implica la creación de significado especial para un determinado producto o servicio, es vital hacerlo distinto en el mercado y particularmente en la categoría de producto que ofrezca (Wells, Moriarty \& Burnett, 2007), algo que debe manejar el Club en cuestión, es reposicionar su marca, dado que estas tienen una vinculación emocional con los consumidores, siempre que sienten que son cumplidas sus promesas, que para el caso sería la consecución de títulos (Kotler \& Keller, 2006). Por tanto, el contacto entre los consumidores y los empleados, así como dentro de la organización, debe ser positivo; las promesas de marca se cumplen, siempre y cuando todos los miembros se compenetren con ese logro (Kotler \& Keller, 2006).

\section{Mercado y Segmentación del Mercado:}

La palabra mercado originalmente significaba el lugar en donde ocurría el intercambio entre vendedor y comprador. Actualmente, un mercado no solamente es considerado como lugar, sino también como un tipo particular de comprador (Wells, Moriarty \& Burnett, 2007).

Puesto que en un mercado determinado existe una variedad muy amplia de clientes posibles, que le impiden a las empresas servir de forma óptima a cada uno de ellos (Thompson, 2005), es necesario identificar los segmentos de mercado, entendidos por este autor como aquellos grupos con características de consumo semejantes, a los que se les puede implementar actividades de mercadeo con diseños particulares para satisfacer sus necesidades,
Prango permitiéndoles un ahorro de tiempo y esfuerzo. En esto concuerdan Kotler \& Armstrong (2003), identificando el segmento de mercado con "un grupo de consumidores que responden de forma similar a un conjunto determinado de esfuerzos de marketing." (p.61)

A su vez, Stanton, Etzel \& Walker (1995), le agregan el deseo y la preferencia entre clientes con estilos de vida semejantes, en donde ya hay cabida no solo para los productos sino los deportes, en ese sentido el Club estará directamente emparentado con su hinchada.

Aunque en principio los estudios sobre segmentación no eran muy tenidos en cuenta; dado que la tendencia era la de producir y vender masivamente (Kotler \& Armstrong, 2007), actualmente las organizaciones que buscan posicionarse competitivamente tienen muy en cuenta la segmentación de mercados para sus productos y servicios.

\section{Niveles de segmentación del mercado:}

Es necesario dividir los clientes potenciales, para conocer las ventajas o desventajas de comercializar, o introducir, un producto al mercado. Por eso es importante realizar una investigación exhaustiva para encontrar el target, 0 segmento meta, que no es más que el grupo de población elegido al cual se le ofrecerá el bien o servicio y, de esta manera, se obtendrá un mejor resultado al momento de generar impacto en la mente del consumidor (Kotler \& Keller, 2006). Las conductas y comportamientos actuales de los consumidores hacen que los expertos en marketing tengan que diseñar planificaciones adecuadas para cada segmento específico, lo cual ha conllevado a la creación de diferentes tipos de marketing entre los cuales tenemos: Marketing viral, marketing de fidelización, marketing médico; marketing deportivo, entre otros (Aljure, 2010).

\section{Marketing Deportivo:}

Actualmente no es abundante la literatura sobre este tipo de marketing. Sin embargo, citando a Sánchez (2003), se le define como la caracterización del Marketing en sus distintas dimensiones, objetivos y su relación con la actividad deportiva, tanto en la toma de decisiones y elaboración de estrategias de posicionamiento, como en la preparación de un plan de marketing, de un producto 0 servicio relacionado con deporte. Por otro lado, Campos (1997), lo define como una herramienta de Marketing que permite relacionar la marca con actividades deportivas, otorgándole, de esta manera, un valor a ésta.

Varios autores, entre ellos Mullín, Ardi y Sutton (1995) y Castellanos (2001), concuerdan en que el marketing deportivo ha desarrollado dos actividades importantes: comercializar productos y servicios a quienes son consumidores de los deportes, es decir los que hacen parte 
del grupo de personas con afinidad hacia determinada disciplina, y servir como vehículo de promoción para productos, marcas y servicios a grupos más allá del deporte. Lo que le ha permitido ser más que una manifestación social, ahora se le considera "un bien, cuya producción, consumo, financiación y gestión responde a criterios de racionalidad económica" (Castellanos, 2001, p.181).

\section{Estrategias del Marketing Deportivo:}

Para Ferrand, Torrigiani \& Camps, (2007) la estrategia más reconocida es el Sponsoring o patrocinio, la cual funciona como forma publicitaria que se integra al marketing con el objetivo comercial de explotación que de una marca a partir de la asociación entre una organización, evento o persona con otra organización. Este acuerdo se hace bajo el imperio de una transacción comercial. Otros autores concuerdan en que la asociación emocional es muy oportuna para el patrocinio, ya que hay conexión entre los consumidores y los equipos deportivos. (Grohs, Wagner y Vsetecka, 2004). Basta con pensar en clubes deportivos como el Real Madrid en España o el Boca Junior en Argentina, los cuales alcanzaron la creación de valor para sus marcas, bajo el concepto de "Resaltar los activos intangibles relacionados con las capacidades de los colaboradores" (Jáuregui, 2008, p.10). De esta forma, el papel de la calidad, fidelización y rentabilidad hacen parte de la llamada "cadena de lealtad" nombrada por Storbacka, Strandvik y Gronroos (1994), la cual sintetiza la importancia de mejorar los vínculos para mantener a los clientes.

\section{Marco Contextual}

A continuación se exponen brevemente las características históricas y deportivas del El Club Deportes Tolima, a fin de ubicar el contexto en el que se presenta la investigación puesto que existen 20 equipos en la primera división del futbol colombiano.

El fútbol profesional colombiano se inició en el año de 1948 según cifras y datos de la Dimayor, año en que se funda este estamento oficialmente; por su parte, el Club Deportes Tolima aparece en el torneo colombiano a partir del año 1955. Su sede de funcionamiento es la ciudad de lbagué, capital del Departamento de Tolima. El estadio donde participa tanto en torneos locales como internacionales, es el Manuel Murillo Toro, el cual fue construido el mismo año de su incorporación al fútbol profesional y tenía una capacidad para 3.000 personas. En la actualidad cuenta con una capacidad para 31.000 asistentes (León, 2003).

La institución, sufre uno de sus mayores golpes deportivos y financieros en el año de 1993, cuando cae a la categoría $\mathrm{B}$; esto debido a los malos resultados, tanto en el campo de juego como en lo administrativo. Durante todo el año de 1994 juega la Copa Concasa, obteniendo excelentes resultados y ganando de nuevo su puesto en la categoría principal del futbol colombiano (Ruiz, 2008). Para diciembre del año 2003, la institución obtiene su mayor conquista alcanzando su primer y único título (tabla 1), en el torneo de fútbol profesional colombiano en la categoría $A$; bajo la dirección del profesor Luis Augusto García, ganaron la final con el Club Deportivo Cali. Este título, los llevó a la participación en el torneo más importante de América del Sur, la Copa Libertadores. Este torneo generó ingresos económicos importantes para la institución y una gran vitrina para mostrar a nivel internacional los jugadores que son del registro del equipo (Ruiz, 2008).

Actualmente la institución cuenta con varias participaciones en torneos internacionales (Figura 2.2), un título o campeonato a nivel nacional y una regularidad en su rendimiento que lo ha posicionado en un lugar importante del escalafón del fútbol profesional colombiano (León, 2005).

\section{Tabla $N^{\circ} 1$.}

Posiciones obtenidas por el Club Deportes Tolima desde 2002 a 2010 en torneos de la Liga Colombiana de Fútbol.

\begin{tabular}{|c|c|c|c|}
\hline \multicolumn{2}{|c|}{ AÑO APERTURA } & $4^{\circ}$ GRUPO & $13^{\circ}$ \\
\hline 2002 & $18^{\circ}$ & CAMPEON & $6^{\circ}$ \\
\hline 2003 & $12^{\circ}$ & $3^{\circ}$ GRUPO & $7^{\circ}$ \\
\hline 2004 & $11^{\circ}$ & $9^{\circ}$ & $5^{\circ}$ \\
\hline 2005 & $2^{\circ}$ GRUPO & SUBCAMPEON & $2^{\circ}$ \\
\hline 2006 & $2^{\circ}$ GRUPO & $3^{\circ}$ GRUPO & $8^{\circ}$ \\
\hline 2007 & $12^{\circ}$ & $2^{\circ}$ GRUPO & $2^{\circ}$ \\
\hline 2008 & $18^{\circ}$ & $4^{\circ}$ GRUPO & $1^{\circ}$ \\
\hline 2009 & $3^{\circ}$ GRUPO & SUBCAMPEON & \\
\hline 2010 & $3^{\circ}$ SEMIFINALISTA & 150 & \\
\hline & $3^{\circ}$ SEMIFINALISTA &
\end{tabular}

Nota: Elaboración propia con datos tomados de los anales del club.

Tabla $\mathrm{N}^{\circ} 2$.

Títulos y posiciones obtenidas por el Club Deportes Tolima desde 1982 en Participaciones Internacionales.

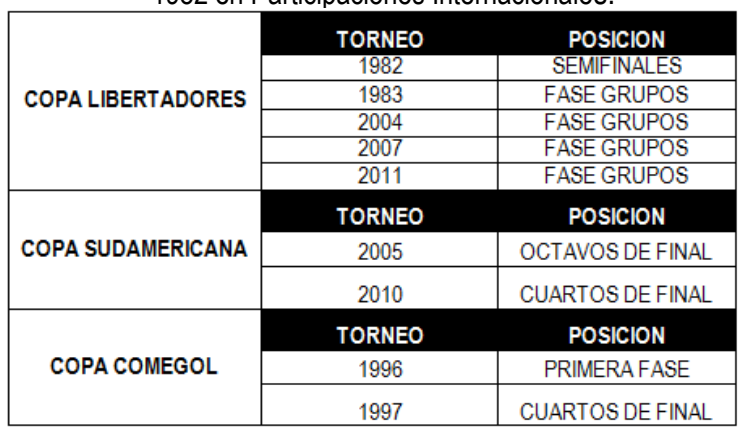

Nota: Elaboración propia con datos tomados de los anales del club. 


\section{EL FÚTBOL COMO VEHÍCULO COMERCIAL DE MARCAS DE BIENES Y SERVICIOS \\ CASO: CLUB DEPORTES TOLIMA}

Andrés Darío Hoyos Arango

Para finalizar, es claro que al ser escasa la teoría en cuanto a marketing deportivo, no hay una definición exacta sobre lo qué es y cuáles son sus alcances. No obstante se pueden unificar pareceres, los cuales apuntan a que son los esfuerzos realizados para comercializar todos los derivados de las diferentes disciplinas deportivas existentes. Los investigadores y trabajadores del marketing deportivo exponen que este modelo estratégico es nuevo en Colombia y que no se explota de la manera más adecuada para sacar los mejores beneficios que este puede dar a las diferentes disciplinas del deporte que se practica en el país.

\section{METODOLOGÍA:}

La investigación se enmarca dentro de las características del paradigma fenomenológico; el cual comprende la realidad como algo dinámico y diverso, cuyo interés va dirigido al significado de las acciones humanas y de la práctica social, dado el caso particular del Club Deportes Tolima. En ese sentido se pretende desarrollar un conocimiento ideográfico con participación comunicativa entre el investigador y los sujetos, jugadores, hinchas y directivos, partiendo desde un análisis holístico, en el que se tienen en cuenta como un todo.

La investigación tendrá un enfoque cualitativo, en tanto se tuvieron en cuenta las características expuestas por los entrevistados, a partir de preguntas de investigación que no necesariamente prueban hipótesis, (Hernández et al., 2003). En el presente caso, se estudiaron grupos pequeños en los cuales se puedo obtener una observación directa y de carácter inductivo. El enfoque cualitativo se enmarca en el paradigma fenomenológico, por tanto, los elementos para la evaluación de las situaciones que conforman la pregunta de investigación se encuentran caracterizados bajo los antecedentes de este paradigma: entrevistas con preguntas abiertas, en profundidad, observación no estructurada, observación participante, y análisis de documentos. La exploración se hará en grupos pequeños de población (directivos, patrocinadores, prensa deportiva, jugadores y aficionados).

Basados en el interaccionismo simbólico, la investigación considerará las siguientes orientaciones metodológicas (Medina, 2009):

La investigación debe estar anclada a la realidad empírica. Las proposiciones teóricas de la investigación dirigida al Club Deportes Tolima, surgirán de los datos obtenidos por experiencia, más que de estudios previos.

Respetar la realidad social, al inicio del estudio sólo se recabaran datos.

La observación, tiene especial significado para obtener información. Se registrará toda conducta y opinión de los directivos, aficionados y las empresas involucradas.

Se debe tomar "el rol del otro". Para obtener un conocimiento desde dentro, se participará activamente en el grupo de estudio, para ver la situación como es vista por los actores, y observando lo que el actor tiene en cuenta.

Captación de la cultura del grupo. Para comprender lo que lo motiva al grupo y sus creencias, según como los individuos del estudio lo entienden; las acciones se articularan no como la suma de acciones individuales, sino como una nueva acción con la participación de individuos (Álvarez-Gayou, 2006).

\section{Diseño de Investigación}

El diseño de la investigación cualitativa se selecciona teniendo en cuenta tres dilemas que experimenta el investigador al momento de obtener, analizar e interpretar la información: la profundidad y extensión del proceso de recolección de datos, por dónde iniciar y terminar la recolección de datos y las unidades de muestreo que se incluyen y excluyen en este proceso (Quintana, 2006).

De acuerdo a lo anterior, en este estudio se propone utilizar 2 tipos de muestreo (Quintana, 2006). El primero consiste en un muestreo por conveniencia porque se requiere seleccionar a los distintos actores fenómeno, de tal forma abarcar el caso de manera holística; y el segundo es un muestreo de caso típico, dado que el objetivo de este estudio es presentar los rasgos más comunes frente al posicionamiento de marca y la fidelización de los aficionados del club Deportes Tolima; para ello, se pretende incluir documentos, entrevistas a directivos, patrocinadores, prensa deportiva, jugadores y aficionados involucrados con el club de manera directa.

Los sujetos u objetos de estudio en esta investigación, son personas que tienen conocimiento y relación con el club Deportes Tolima, especialmente radicados en la ciudad de Ibagué, Colombia. 


\section{RESULTADOS Y DISCUSIÓN:}

Las unidades de análisis en este estudio, es decir los casos 0 elementos sobre los que se recogerá información corresponden a 6 personas que cumplan con el siguiente perfil:

1. Gerente deportivo del Club Deportes Tolima: Sr. Ricardo Salazar.

2. Enertolima, Patrocinador del Club Deportes Tolima: Sr. Wilton Ariel Reyes.

3. Mercacentro, Patrocinador del Club Deportes Tolima: Sra. Cristina Ortiz.

4. Periodista Deportivo Radio Todelar de Cali: Sr. Marino Milán.

5. Aficionado del Club Deportes Tolima: Sr. Heimar Espinoza

6. Análisis de documentos extraídos tanto de fuentes primarias, como de secundarias.

Teniendo en cuenta que "en la indagación cualitativa, el tamaño de la muestra no se fija a priori" (Newman 2009 citada por Hernández, 2010, p.395), pues esta sólo se conocerá al final cuando se dé una "saturación de categorías", es decir cuando los casos adicionales no agreguen información nueva, tentativamente se plantea obtener una muestra que incluya al menos a una persona que corresponda a cada uno de los perfiles de los sujetos de estudio, descritos anteriormente.

\section{Técnicas o Instrumentos:}

En esta investigación se utilizarán como técnicas: el análisis de documentos y la entrevista en profundidad. Del análisis de documentos se retoma lo expuesto por Galeano (2007) "en la investigación documental, todos los textos pueden ser entrevistados mediante las preguntas que guían la investigación y se los puede observar con la misma intensidad con que se observa un evento 0 un hecho social". (p.34), según esta autora, los documentos se agrupan de acuerdo a su intencionalidad, así se realizó con la documentación teórica expuesta anteriormente. En cuanto a la entrevista en profundidad, esta se toma como abierta, flexible, dinámica y no estructurada; sigue el modelo de conversación entre iguales, en vez de ser un intercambio de preguntas y respuestas. Se inicia al establecer rapport con el informante, para ello se formulan preguntas no directivas, con el fin de aprender lo que es importante para el entrevistado, esto ayuda a familiarizarse con el fenómeno de estudio (Hernández, 2010).
Interpretación de los datos

Problemática del Club Deportes Tolima: En esta categoría, se diagnostica la realidad que vive el club, como son la falta de compromiso de la afición, la comercialización de los jugadores, falta de estrategias de marketing agresivas y los patrocinios.

Compromiso de la Afición: Al ser lbagué una ciudad pequeña, que no cuenta con buenas vías de acceso y una infraestructura comercial desarrollada, el porcentaje de desempleo es alto, lo que influye en que el aficionado no cuente con recursos suficientes y prefiera ver los partidos en casa sin costo, versus asistir al estadio, donde no se puede participar de un momento familiar, porque el ingreso de los niños está prohibido. Falta tener mayor apoyo de la afición, más apoyo de la empresa local y sentido de pertenencia; el número de aficionados está en un promedio de 4.000 personas. El club ha trabajado poco en mercadeo, lo que dio como resultado que la afición "envejeció" y los jóvenes se han cambiado a otros equipos.

Resultados en Torneos: En los últimos 5 años ha sido protagonista en los torneos locales, ha tenido corta participación en los torneos internacionales. En opinión de Heimar Espinoza, el club debe aprovechar no tiene competencia en la región, y seguir manteniendo buenos resultados en los torneos; ya que el mejor marketing son los resultados.

Estrategias de Marketing: Según Marino Millán y Heimar Espinoza, se deben crear estrategias convincentes para que la marca Deportes Tolima alcance sus metas y objetivos; el mercadeo y la publicidad juegan un papel fundamental para que se conozcan los beneficios que se tiene siendo sponsor e hincha del Club Deportes Tolima. Además, se debería buscar una marca de prenda que venda y tenga más reconocimiento, así como una tienda donde se consigan implementos deportivos del club (vasos, relojes, esferos, etc.). Desde el principio de cada temporada, debe implementarse un plan de mercadeo que ayude a que la institución tenga un norte hacia dónde dirigirse en lo administrativo, financiero y económico.

Problemas Administrativos: El club debe, según Ricardo Salazar, tener un mejor manejo administrativo, más diligencia en los trámites internos y darle más trayectoria y 


\section{EL FÚTBOL COMO VEHÍCULO COMERCIAL DE MARCAS DE BIENES Y SERVICIOS CASO: CLUB DEPORTES TOLIMA}

Andrés Darío Hoyos Arango

permanencia a los procesos. A criterio Marino Millán, el Club debe empezar a pensar como una empresa y club (con sede y escuela deportiva), no como un grupo de trabajo; así como menciona Cristina Ortiz, los hinchas deben tener contraprestaciones con el equipo.

Cadena de Posicionamiento:Está integrada por subcategorías: prensa deportiva, aficionados, empresarios e imagen internacional.

Prensa Deportiva: Marino Millán menciona la importancia que esta prensa tiene para el club, pues beneficia comercialmente a los involucrados; ayuda a que el equipo tenga más reconocimiento fuera y dentro del país. La televisión es una opción interesante para la institución; en el exterior el Deportes Tolima es poco conocido y la TV sirve de vitrina para que clubes del exterior se fijen en los jugadores. El club tiene el apoyo de las emisoras locales y empresarios que ven el beneficio de apoyar al Club Según Cristina Ortiz y Wilton Reyes; se debe trabajar para acercarse más a ellos y a otros medios de comunicación, la radio es fundamental, al ser el medio más barato para invitar a los oyentes a que asistan a los partidos.

Aficionados: Marino Millán expresa: hoy ser hincha de un equipo extranjero, es tan fácil como de uno nacional.

Empresarios: Los empresarios que trabajan con el Club son en su gran mayoría entidades públicas (como la industria de Licores del Tolima con su aguardiente Tapa Roja), empresas regionales, nacionales e internacionales. La Alcaldía de Ibagué y la Gobernación representan un apoyo importante, sin el cual el Club no podría seguir compitiendo. Cada vez hay más empresas que ven al Deportes Tolima como un buen canal para posicionar su marca, lo cual es positivo para el club ya que se logra una recordación. Según Wilton Reyes hay que trabajar en las relaciones entre los jugadores, el personal técnico y los patrocinadores.

Imagen Internacional: En la clasificación de la FIFA en el 2011, el Club se encontraba en el puesto 26. En estos momentos, menciona Cristina Ortiz, cuenta con un pobre reconocimiento en el exterior, por sus buenas participaciones y empieza a ser reconocido, por la venta de jugadores a equipos extranjeros. .

Comunicación Integral con sus sub-categorías comunicación directa, canales de comunicación, patrocinios, participación en eventos, promoción y programas de fidelización.
Comunicación Directa: Existe un canal de comunicación directa, entre el área de mercadeo y el gerente deportivo con los empresarios e industrias del sector.

Canales de Comunicación: Entre los canales de comunicación que utiliza el club figuran las bases de datos que se obtienen por medio de la boletería; actualmente busca nuevos clientes a través de las redes sociales y telemarketing. Según Marino Millán Se debe trabajar más con las radios y los periodistas que venden los espacios publicitarios en la transmisión de los partidos y en la pauta televisiva. Los espacios en las emisoras son muy reducidos o los comentarios muy efímeros, los cuales no causan recordación a nivel nacional. Se debe implementar una estrategia de segmentación para sacar un mayor provecho al vender las partes del uniforme y las vallas en el estadio.

Patrocinios: El equipo cuenta con un departamento de marketing encargado de contactar empresarios para que sean patrocinadores, su política es no manejar exclusividad. La gerencia de mercadeo viene logrando resultados positivos, los patrocinadores actuales son la Alcaldía, la Gobernación, empresas privadas de la región que son líderes en el mercado, empresas nacionales y otras internacionales. Siendo el principal ingreso los patrocinadores, hay que salir según Wilton Reyes, y Heimar Espinoza, a buscar más, cuyos objetivos sean el reconocimiento o quieran posicionar su marca y cuenten con el presupuesto para hacerlo; siempre generando percepción positiva y que la gente perciba el respaldo social. Según Ricardo Salazar, vender un intangible no es fácil, se busca la manera de que el empresario perciba el beneficio y retorno de la inversión. Además se requiere de una campaña deportiva que se justifique en los primeros lugares, una nómina competitiva y crear afinidad con los aficionados, es indispensable estar apoyado por marcas reconocidas a las cuales se les ofrece espacios para ser utilizados con dummie, material POP, avisos en el estadio y la participación en la revista Corazón Pijao. Sus patrocinadores actuales son, , Mercacentro (Cadena de Supermercados), Multicentro (Centro Comercial), Comfenalco (Caja de compensación familiar), Aguardiente Tapa Roja (Licorera del Tolima), CP Company (Prendas de vestir), Enertolima (Electrificadora), Lubricantes Gulf.

Promoción: Con campañas de mercadeo, se busca despertar más empatía del hincha hacia el Club; como ofrecer productos deportivos con la marca, que la camiseta no sea lo único que se vea; el mercadeo de ingreso para llevar más gente al estadio, con el gancho por una boleta entran dos; y posicionar la revista del Club. Cristina Ortiz menciona: siendo líderes del torneo, hay mayores posibilidades de que tanto empresas como hinchas apoyen con más fuerza la institución, la vinculación de jugadores 
competitivos al equipo y con participaciones más representativas en torneos internacionales.

Programas de Fidelización: Anteriormente la gente acompañaba más al equipo y los patrocinadores aportaban más, pero los tiempos han cambiado; se debe trabajar, según Wilton Reyes en mejorar el espectáculo en el estadio, creando atractivos que motiven a los aficionados. Se está trabajando en la implementación del programa "Hincha más fiel" desarrollado por la firma antioqueña Servicios Integrales S.A., mediante tarjetas de fidelización.

Asociatividad: En marketing deportivo, menciona Cristina Ortiz, todos los equipos europeos como Barcelona, el Real Madrid, el Manchester United son interesantes, aquí en Sudamérica destaca el Boca Juniors y el River Plate de Argentina, quienes tienen una estructura organizativa que ayuda a la consecución tanto de resultados financieros como deportivos. Tienen un concepto claro del marketing.

Estrategias de Posicionamiento: Las diferentes estrategias que lleva a cabo el Club, son impuestas por el Sr. Camargo Presidente del Club, quien tiene la visión, toma las decisiones y da las directrices, a pesar de no ser un experto en el tema según Marino Millán. Se detecta la falta de organización como un verdadero club y empresa. A pesar de tener un gerente de mercadeo, quien ha trabajado y ha logrado una mejora comercial, falta contratar personal especializado en marketing deportivo.

Marketing de Relaciones: La TV, la radio y la publicidad escrita, son medios fundamentales para promocionar los partidos y los jugadores mejor rankeados. Los equipos venden de acuerdo a sus aficiones y buenas participaciones. Hay que tener un concepto claro de empresa según Heimar Espinoza, buscar inversionistas con alto capital e identidad con la gente que apoyen al equipo.

\section{CONCLUSIONES:}

Más allá del respaldo en el estadio con su asistencia a los partidos y el apoyo constante al equipo, el aficionado genera un impacto importante en el modelo de marketing, pues este se convierte en el consumidor potencial del equipo como marca e igualmente de las vinculadas a este. Así un equipo con un mayor número de aficionados tiene un mayor poder de negociación con las empresas y una mayor posibilidad de obtener mejores ingresos. Falencia latente del Club Deportes Tolima el cual cuenta con una afición no mayor a los 4.000 seguidores en promedio por partido actuando como local, por lo cual es considerado equipo pequeño por la poca afición que lo acompaña; a pesar de ser un equipo que tiene 57 años de ser fundado y contar con muy buenas campañas como la década de los 80 y en el 2003, donde quedó campeón y con un buen desempeño hasta la fecha.

Las empresas buscan clubes que apoyen y reflejen los valores de sus marcas. Por esta razón, la búsqueda de alianzas estratégicas para las empresas que intentan generar relaciones de largo plazo, sólo se logran con la buena gestión de un modelo claro en marketing deportivo.

Las líneas de negocio, con mayor impacto en un modelo de marketing deportivo actualmente son los contratos de transmisión de partidos, los patrocinios comerciales y la asistencia al estadio. En Colombia, los mayores ingresos los generan las dos primeras líneas, en función del reconocimiento y los logros obtenidos por el equipo. Sin embargo, también se pueden crear otras alternativas de líneas de negocio, principalmente enmarcadas en la formación de jugadores y divisiones menores que promuevan una futura venta de jugadores a otros clubes o ligas extranjeras y la comercialización de su marca en productos deportivos y souvenirs.

Cada vez es mayor la competencia de la televisión por cable; esto ha generado un incremento en las ofertas a los clubes colombianos de fútbol. La variable éxito deportivo, es un factor que incide directamente en el poder de negociación de los clubes, ante las cadenas de televisión por el torneo local y también les da la posibilidad de obtener ingresos de cadenas internacionales por la transmisión de los partidos, de los torneos, y de las copas Libertadores de América y Sudamericana.

En el modelo de marketing deportivo, los grandes clubes de fútbol cuentan al menos con un estadio propio, una sede social y una estructura de división de menores; además de un plantel con figuras reconocidas y una dirigencia profesional. En Colombia, la gran mayoría de los equipos no tienen esta infraestructura y en ello radica uno de los principales motivos por los que el fútbol se sigue viendo sólo como un deporte y no como una oportunidad de negocio. Situación real del Deportes Tolima, que no se comporta como verdadero club, si no como un grupo de trabajo o un equipo de fútbol.

El club Deportes Tolima no se ha encargado de diseñar planes de mercado por medio de los cuales se busque generar la fidelización de todo su target (Hinchas y empresas) en el largo plazo. Esto es fundamental para el sostenimiento y viabilidad de una institución deportiva, ya que a medida que esta cuenta con este soporte, puede ser más competitiva tanto en lo administrativo, como en lo deportivo. 


\section{EL FÚTBOL COMO VEHÍCULO COMERCIAL DE MARCAS DE BIENES Y SERVICIOS CASO: CLUB DEPORTES TOLIMA}

Recomendaciones: De acuerdo a lo expresado en las conclusiones de este trabajo, se propone tener en cuenta las siguientes recomendaciones:

Para incrementar el valor de marca el Deportes Tolima, deberá realizar una evaluación previa de la situación en la que se encuentran respecto de cada una de las variables y

luego plantear objetivos reales que permitan iniciar el diseño de un plan de negocios que contenga un modelo en marketing deportivo adaptado a las necesidades del Club. Con estrategias que conlleven a mejorar el posicionamiento y valor de su marca para un mejor desarrollo económico, comprometiéndose en un proceso a largo plazo.

Invertir en las divisiones menores, con personal capacitado y profesional que asegure al Club una producción de futbolistas para el equipo principal, mercado nacional e internacional. Esto se podría considerar como un medio para generar un vínculo más cercano con el aficionado y la región. Se puede complementar con un proyecto de desarrollo de un centro de alto rendimiento "Deportes Tolima" para niños. El objetivo es poder aportar al perfil psicosocial del jugador de fútbol desde la niñez y, de esta manera, asegurar que jóvenes con talento desarrollen su potencial natural de forma integral (físico, educativo y psicológico). Esto repercutirá socialmente en el país, al incluir a jóvenes de cualquier estrato y condición social con aptitudes para el fútbol, y económicamente para el Club, con el retorno de la inversión al vender jugadores a clubes nacionales o ligas extranjeras.

Profesionalizar su plana administrativa y equipo de jugadores. En este sentido, la capacitación permanente en cursos de actualización de administración, marketing y recursos humanos, etc. logrará que el club Deportes Tolima y su grupo de colaboradores junto con sus stakeholders se sientan ubicados en una categoría más segura y de crecimiento tanto profesional, intelectual y personal. Adicionalmente, el Club debe mantener un equipo competitivo con jugadores emblemáticos, para que este, sea atractivo al aficionado. Se debe buscar establecer alianzas estratégicas con clubes de éxito en el exterior, lo que permitirá hacer un benchmarking que permita adquirir e identificar cuáles son las variables de éxito para poder desarrollarlas en el Deportes Tolima.

Para incrementar la asistencia al estadio "Manuel Murillo Toro", el Club Deportes Tolima debe aplicar estrategias de fidelización. A través de las redes sociales y paginas interactivas. El objetivo es generar un vínculo directo entre la afición y el club por medio de la creación de bases de datos; que permitan interactuar, intercambiando puntos de vista y participando directamente en el desarrollo de la institución deportiva, concientizándolos sobre que es necesaria su participación incondicional para incrementar los ingresos y lograr la estabilidad del club. Esto se puede realizar por medio de un programa de Hospitality "que la FIFA a partir del mundial de Sudáfrica en el año 2010 creó", en donde se dejan acercar los hinchas a los equipos o selecciones de fútbol profesional. El club Deportes Tolima puede encontrar una oportunidad, en donde se abren las puertas para que los hinchas del equipo, por medio de una inversión al club pueda compartir el entrenamiento, almorzar con los jugadores y directivos, entrar al camerino el día del partido en un palco especial pueda compartir con sus más allegados.

\section{REFERENCIAS:}

Aljure A. (2010). Marketing en el futbol. Obtenido el 25 de marzo del 2012. Grupo Dir. Disponible en: http://www.grupodircom.com/tapas/edicion/765$\underline{\text { revista-dircom-91-marketing.html }}$

Álvarez-Gayou J. (2006). Cómo hacer Investigación Cualitativa: Fundamentos y Metodología. México: Paidós.

Campos C. (1997). Marketing y Patrocinio Deportivo. Barcelona: GPE Colección Gestión Deportiva.

Castellanos P. (2001). Análisis de la demanda y economía del deporte. En Otero Moreno, J. M. (director), Incidencia Económica del Deporte, (pp. 181-189). Málaga: Instituto Andaluz del Deporte.

Ferrand A., Torrigiani L., Camps Povill A., (2007). Gestión del Sponsoring Deportivo. España: Paidotribo, 1ra Edición.

Galeano, M.E (2007). Estrategias de investigación social cualitativa: el giro en la

Mirada. Editorial: Medellín, La carreta editores.

Gómez, A. (2009). Publicación Portafolio. Redacción Economía y Negocios. Obtenido el 23 de enero de 2009. Disponible en: http://www.portafolio.co/

González, E. (2010). Los factores de competitividad: el efecto grupo. Revista D y 0. 3. p.140. Disponible en: http://www.revistadyo.com/index.php/dyo/article/vi ewFile/140/140 
Grohs, R., Wagner, U. \& Vsetecka, S. (2004). Assessing the Effectiveness of Sport

Hernández R., Fernández C., Baptista M. (2010). Metodología de la Investigación. México: Mc Graw Hill Educación, 5ta Edición.

Jáuregui, K. (2008). La creación de valor. Redacción El Comercio. Obtenido el 10 de abril del 2012. Disponible

en: http://elcomercio.pe/edicionimpresa/html/2008-0708/la-creacion-valor.html

Jiménez, H. (2011) Estos son los equipos morosos del fútbol colombiano. Nuevo Estadio. Obtenido el 03 de marzo del 2012, Disponible en: http://www.nuevoestadio.com/2011/01/13/estosson-los-equipos-morosos-del-futbol-colombiano/

Kotler, P \& Armstrong, G. (2003). Fundamentos de marketing. Barcelona.: Ed Pirámide. p.448.

Kotler, P. (2001). Fundamentos de Mercadotecnia. México: Prentice Hall, $8^{a}$ Edición.

Kotler, P., Keller K. (2006) Dirección de Marketing. México: Pearson Education, 12va. Edición.

López, C.2003. Redes Empresariales. 1a ed. Trujillo, Perú. MINKA. p.216.

Medina A. (2009) Diseños Cualitativos. Tesis de grado. Universidad Autónoma de Baja California. Disponible en: http://www.slideshare.net/guest8937b2/diseoscualitativos-de-investigacin

Molina, G \& Aguiar F. (2003). Marketing Deportivo. Buenos Aires, Argentina: Ed. Norma, p. 245.

Mullin, B., Ardí, S., Sutton, W. (1995). Marketing deportivo. Barcelona: Paidotribo.

Porter, M. (1990). The competitive advantage of nations. Harvard Business Review. Disponible en: http://dl1.cuni.cz/pluginfile.php/50387/mod_resour ce/content/0/Porter-competitive-advantage.pdf

Quintana, A. (2006). Metodología de Investigación Científica Cualitativa. En Quintana, A. y Montgomery, W. (Eds) Psicología: Tópicos de actualidad. Lima: UNMSM (pp. 56-59).

Rodríguez, M. (2009). Ya era hora de intentar algo. Obtenido el 29 de abril del 2012. Disponible en: http://www.deportestolima.com/noticias/985-iyaera-hora-de-intentar-algo
Ruíz G. (2008). La Gran Historia del Fútbol Profesional Colombiano. Ediciones Dayscript.

Sánchez P. (2003), Marketing Deportivo. Buenos Aires, Argentina: Editorial Norma, $1^{\mathrm{a}}$ Edición.

Stanton E. (1994). Fundamentos de Marketing. México: Mc Graw Hill, ga Edición.

Stanton, W., Etzel, M., Walker, B. (1994). Fundamentos de Marketing. México: Mc Graw Hill, ga Edición.

Storbacka K.; Strandvik T.; Gronroos C. (1994), «Managing customer relationships for profit: the dynamics of relationship quality». International Journal of Service Industry Management. Sponsorships An Empirical Examination. Schmalenbach Business Review, 56(2), 119-138.

Thompson I. (2005, Agosto 01). El Segmento de Mercado. Promonegocios.net. Obtenido el 01 de Abril del $2012 . \quad$ Disponible en: http://www.promonegocios.net/mercadotecnia/seg mento-mercado-definicion-concepto.htm

Torres, R. (2009). Tolima 7 días. Obtenido el 18 de marzo del 2012. Disponible en: http://www.deportestolima.com/noticias/1147deportes-tolima-atraviesa-nuevamente-por-unadificil-situacion-economica-

Wells W., Moriarty S., Burnett J. (2007). Publicidad Principios y Práctica. México: Editorial Pearson Prentice Hall, 7ma Edición: p. 33. 